\title{
A TRANSITION RADIATION DETECTOR WHICH FEATURES ACCURATE TRACKING AND dE/dx PARTICLE IDENTIFICATION*
}

\author{
E. O'Brien, D. Lissauer, S. McCorkle, V. Polychronakos, H. Takai \\ Brookhaven National Lab, Upton, New York, 11973 \\ C.Y. Chi, S. Nagamiya, W. Sippach, M. Toy, D. Wang, \\ Y.F. Wang, C. Wiggins, W. Willis \\ Columbia University, New York, New York,10027 \\ V. Cherniatin, B. Dolgoshein \\ Moscow Institute of Physics and Engineering, Moscow, Russia \\ M.Bennett, A.Chikanian† S. Kumar, J.T. Mitchell, K. Pope \\ Yale University, New Haven, Connecticut, 06511 \\ BNL- -48201
DE93 006391
}

\section{Abstract}

We describe the results of a test run involving a Transition Radiation Detector that can both distinguish electrons from pions with momenta greater than $0.7 \mathrm{GeV} / \mathrm{c}$ and simultaneously track particles passing through the detector. The particle identification is accomplished through a combination of the detection of Transition Radiation from the electron and the differences in electron and pion energy loss $(\mathrm{dE} / \mathrm{dx})$ in the detector. The $\mathrm{dE} / \mathrm{dx}$ particle separation is most efficient below $2 \mathrm{GeV} / \mathrm{c}$ while particle ID utilizing Transition Radiation is effective above $1.5 \mathrm{GeV} / \mathrm{c}$. Combined, the electron-pion separation is better than $5 \times 10^{2}$. The single-wire, track-position resolution for the TRD is $\sim 230 \mu \mathrm{m}$.

\section{Introduction}

Experiments being designed for the Relativistic Heavy Ion Collider (RHIC) at Brookhaven National Laboratory will need to distinguish electrons from pions to better than $0.1 \%$ over a momentum range of $0.1 \mathrm{GeV} / \mathrm{c}$ to $8.0 \mathrm{GeV} / \mathrm{c}$. The particle multiplicity will be high $(\mathrm{dN} / \mathrm{dy} \geq 1000)$ but the event rate for central $\mathrm{Au}-\mathrm{Au}$ collisions will be relatively modest $(1-10 \mathrm{kHz})$. Transition Radiation Detectors (TRD's) are well suited for separating electrons from pions in a high multiplicity environment within a colliding beam geometry. By conducting a test run with a TRD prototype we have been able to quantify many of the RHIC TRD performance parameters.

We have designed a TRD for inclusion in a RHIC experiment that allows electron-pion separation utilizing both $\mathrm{TR}$ and $\mathrm{dE} / \mathrm{dx}$ information, while simultaneously tracking charged particles w: th good position accuracy[1]. A prototype of this detects. has been tested in a secondary beam

\footnotetext{
-Work supported in part by the U.S. Department of Energy

i Permanent adiress: Lebeder insitutie of Phjsics, Mescow, Russia
}

of the Alternate Gradient Synchrotron at Brookhaven National Laboratory. In this test run we have determined the $e / \pi$ separation of our prototype over a momentum range of $0.7 \mathrm{GeV} / \mathrm{c}-4.0 \mathrm{GeV} / \mathrm{c}$, evaluated a variety of potential radiator materials for the TRD and measured the TRD tracking resolution.

\section{The Test Run}

The TRD used in our test run was a modified version of the NA34 TRD[2]. It consisted of four wire chambers interleaved with radiator material. Each wire chamber was a xenon-filled Time-Expansion Chamber with a $1 \mathrm{~cm}$ drift space and a proportional region of $8 \mathrm{~mm} \times 2.5 \mathrm{~mm}$ per anode wire (Fig. 1). A single wire chamber consisted of a $10 \mu \mathrm{m}$ aluminized mylar front entrance window that served as both a cathode to the chamber's drift section and a gas barrier, a plane of vertical cathode wires located at the beginning of the chamber's proportional region, a plane of horizontal, $25 \mu \mathrm{m}, \mathrm{Au}-\mathrm{W}$ anode wires spaced $\sim 2.5 \mathrm{~mm}$ apart and cathode strips etched into a rear aluminized mylar window. Each of the four wire chambers was separated from the next by $16.8 \mathrm{~cm}$ in the z-direction (beam direction). The radiator material of the TRD consisted of either $10 \mathrm{~cm}$ thick blocks of polyethylene foam or 120 layers of $12 \mu \mathrm{m}$ polypropylene foils spaced by $800 \mu \mathrm{m}$. Several radiator materials were tested. The RHIC TRD and prototype differ in the length of the drift region, cathode orientation and number of detector planes. The prototype contains 4 planes of radiator-detector, a $1 \mathrm{~cm}$ drift space and cathode strips perpendicular to the anode wires, while the RHIC TRD will have 8 detector planes, a $3 \mathrm{~cm}$ drift space, and cathode strips oriented for stereo readout.

The electronics chain on each anode wire or cathode strip consisted of a preamplifier, a shaping amplifier and a 6-bit Flash ADC clocked at 24.5 nsec. The FADC had a memory depth of 256 bins or $6.25 \mu \mathrm{sec}$. The digitized 

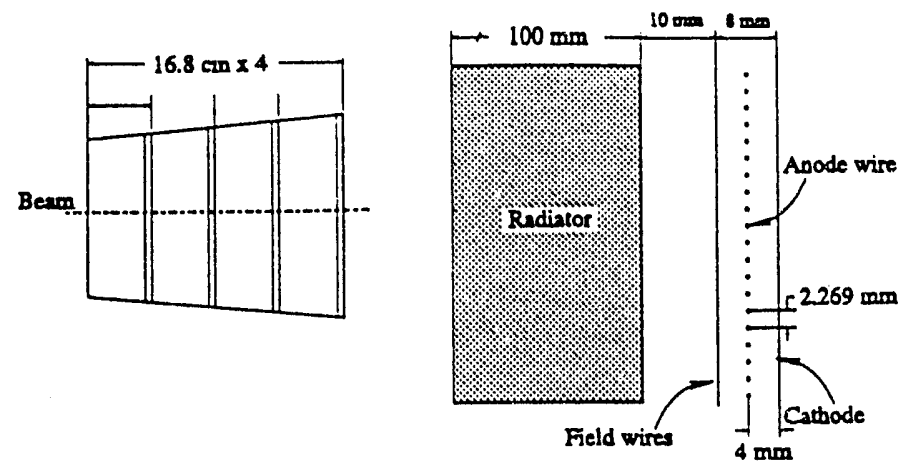

Figure 1: Schematic of Test TRD

FADC signal was read through a VME DRIIW into a VME crate that also received CAMAC data from detector elements in the beamline (Fig. 2). The whole data aquisition system was controlled by a Macintosh running Mac-UA1 software from CERN.

The test run took place at the A2 beamline of the AGS at BNL and used a secondary beam containing $e^{-}$'s, $\pi^{-}$'s and $K^{-1}$ s. The beamline was instrumented with two beam defining scintillators $\mathrm{S} 1$ and $\mathrm{S2}$, a veto counter S3, two Cerenkov counters $\mathrm{C} 1$ and $\mathrm{C} 2$, and a block of Pb-glass 10 radiation lengths deep (Fig. 3). The data were taken either with a minimum bias trigger ( $S 1 . S 2$ ), or an electron trigger $(S 1 \cdot S 2 . C 1 . C 2)$, where both the Cerenkov counters were set for $e / \pi$ discrimination. The $e / \pi$ ratio in the beam ranged from $0.5 \%$ to $20 \%$ depending on the beam momentum.

\section{Radiator Tests}

The number of transition radiation photons produced for 6 different polyethylene foam radiators, and a polypropylene foil radiator, was measured. Each radiator was $10 \mathrm{~cm}$ ihick. Our goal was to determine the absolute transition radiation production of each radiator and the production of TR photons per radiation length of material. The cell sizes and densities of our test radiators are listed in the following table.

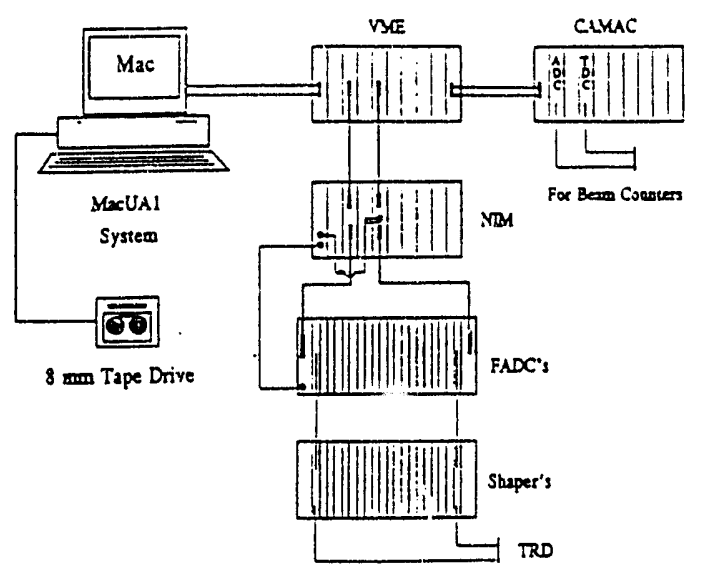

DAQ SYSTEM FOR TRD BEAM TEST

Figure 2: Test run electronics chain

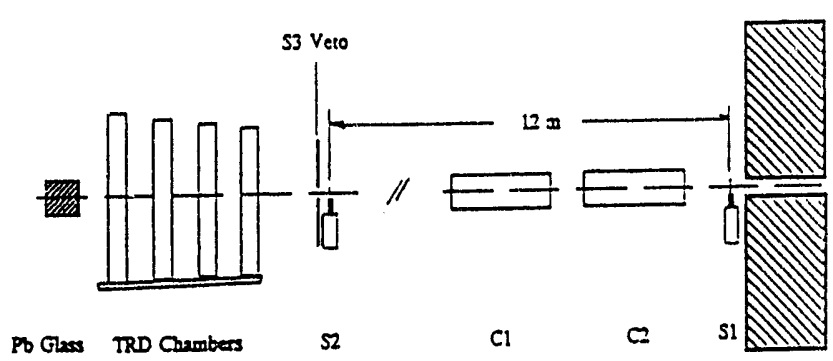

Figure 3: Overview of Test Beamline Set-up 


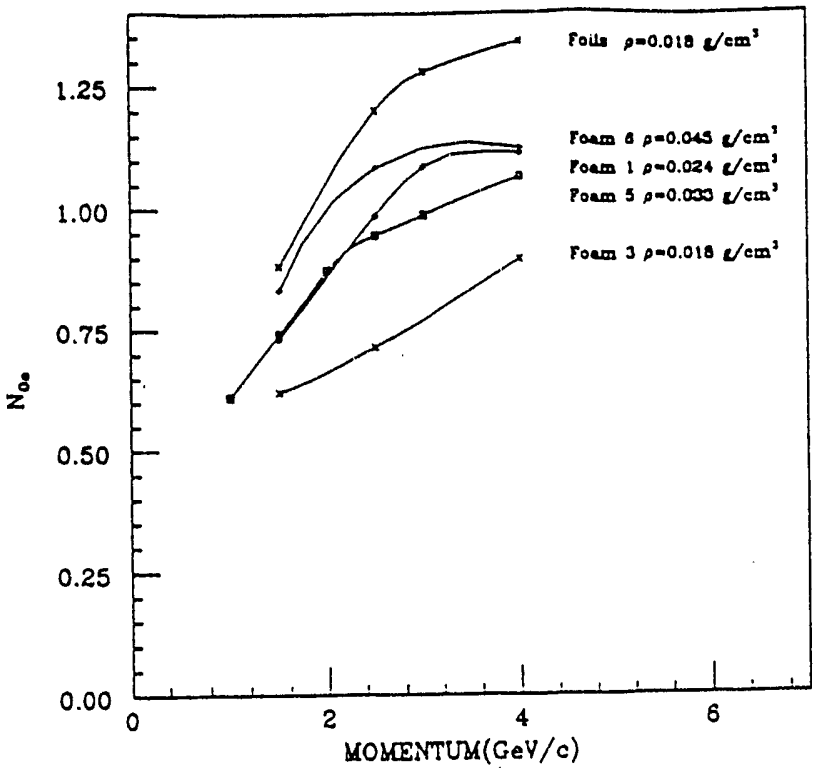

Figure 4: Number of photon clusters over threshold per 10 $\mathrm{cm}$ layer of radiator. The data is from electrons at different momenta.

\begin{tabular}{|c|c|c|}
\hline Radiator Type & Density & Cell Size or Foil Gap \\
\hline Foil & $0.018 \mathrm{gm} / \mathrm{cm}^{3}$ & $800 \mu \mathrm{m}$ \\
Foam 1 & $0.024 \mathrm{gm} / \mathrm{cm}^{3}$ & $800 \mu \mathrm{m}$ \\
Foam 2 & $0.044 \mathrm{gm} / \mathrm{cm}^{3}$ & $1000 \mu \mathrm{m}$ \\
Foam 3 & $0.018 \mathrm{gm} / \mathrm{cm}^{3}$ & $600 \mu \mathrm{m}$ \\
Foam 4 & $0.024 \mathrm{gm} / \mathrm{cm}^{3}$ & $200 \mu \mathrm{m}$ \\
Foam 5 & $0.033 \mathrm{gm} / \mathrm{cm}^{3}$ & $600-700 \mu \mathrm{m}$ \\
Foam 6 & $0.045 \mathrm{gm} / \mathrm{cm}^{3}$ & $200-250 \mu \mathrm{m}$ \\
\hline
\end{tabular}

Both electron and pion data were taken for each radiator at three or more momentum settings. The FADC data from each event was scanned for photon clusters. A photon cluster was defined as $E \geq 4.5 \mathrm{keV}$ deposited inside the xenon gas chamber in a volume 0.5 to $1.5 \mathrm{~mm}$ in diameter. We called this an identified $T R$ fhoton. The size of the cluster seen in our detector is determined by a combination of the initial electron cluster size due to the absorption of the X-ray, the gas diffusion over the drift distance, and the rise time of our shaping amplifier. The energy scale of the clusters wore set by normalizing the FADC pulse heights to an ${ }^{85} \mathrm{Fe}$ source. The relative normalization of each wire was obtained with pulser calibration runs that involved injecting a known amount of charge into the input of each preamplifier. The ${ }^{55} \mathrm{Fe}$ normalization was performed at least once every eight hour running period. The pulser calibration was found to be consistent over many days.

The mean numbers of TR photons, as defined above, were calculated for each radiator at each beam momentum and are shown in figure 4 . We can normalize the mean number of TR photons produced per radiation length of the radiator material (Fig. 5) and see that on average the foil radiator produces $50 \%$ more TR photons than the foam adiators. It should be noted that pure pion data

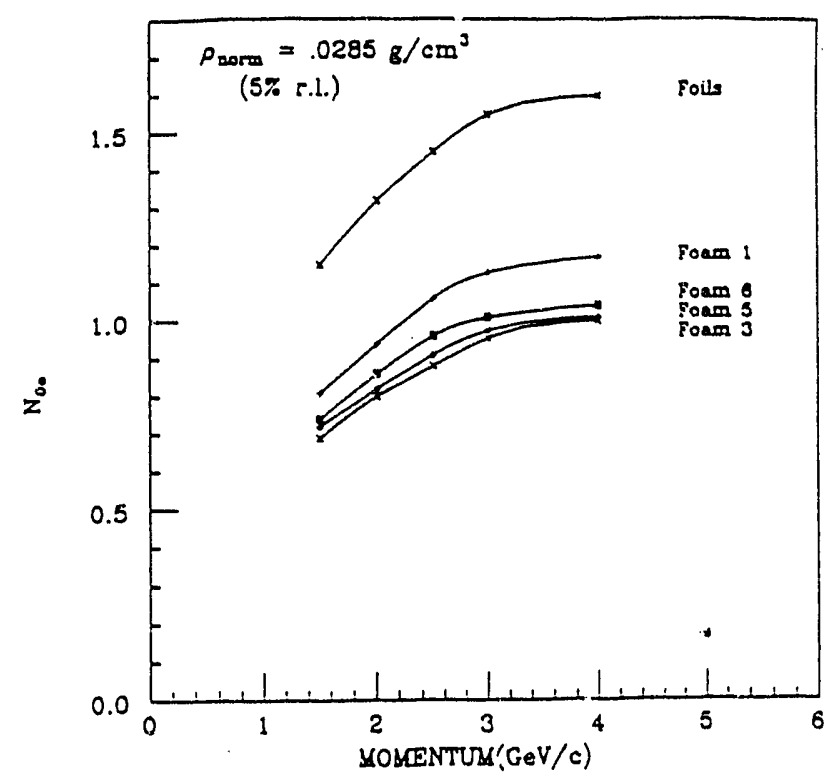

Figure 5: Number of photon clusters produced by electrons in 1 layer of radiator normalized where 8 layers of radiator $=5 \%$ radiation length.

also show pions producing what are apparently TR photons, although at a much lower rate than electrons. Large energy clusters in pion data come from $\delta$-rays, and it is this background that ultimately limits the ability of a TRD to separate electrons from pions.

\section{Transition Radiation $e / \pi$ Separation}

Once transition radiation can be identified in our detector the $e / \pi$ rejection can be determined by histogramming the TR photon cluster distributions for electrons and pions traveling through many layers of the TRD. The TRD that we have designed for RHIC experiments will include 8 layers of radiator and gas detector, so to calculate $e / \pi$ separation with data from our 4 layer test detector we have paired consecutive electron or pion events. We believe that summing pairs of tracks is an accurate method because backgrounds that degrade pion rejection, such as $\delta$-rays accompanying pions through 8 planes of the TRD, are either rare or readily identified by the tracking festures of the detector.

Large statistical samples of both electrons and pions were accumulated at a few momenta for one particular foam radiator. Additional data were taken with the foil radiator for normalization purposes. Large statistics are required to determine $e / \pi$ separation to the $10^{-3}$ level and beyond.

In the analysis we accepted events that contained only single tracks, summed charge from neighboring wires to reduce diffusion effects, and employed threshold cuts as outlined in the previous section. We also utilized a cut called ti.e intelligent threshold, which applies a sliding ADC threshold that moves from low to high as the clus- 


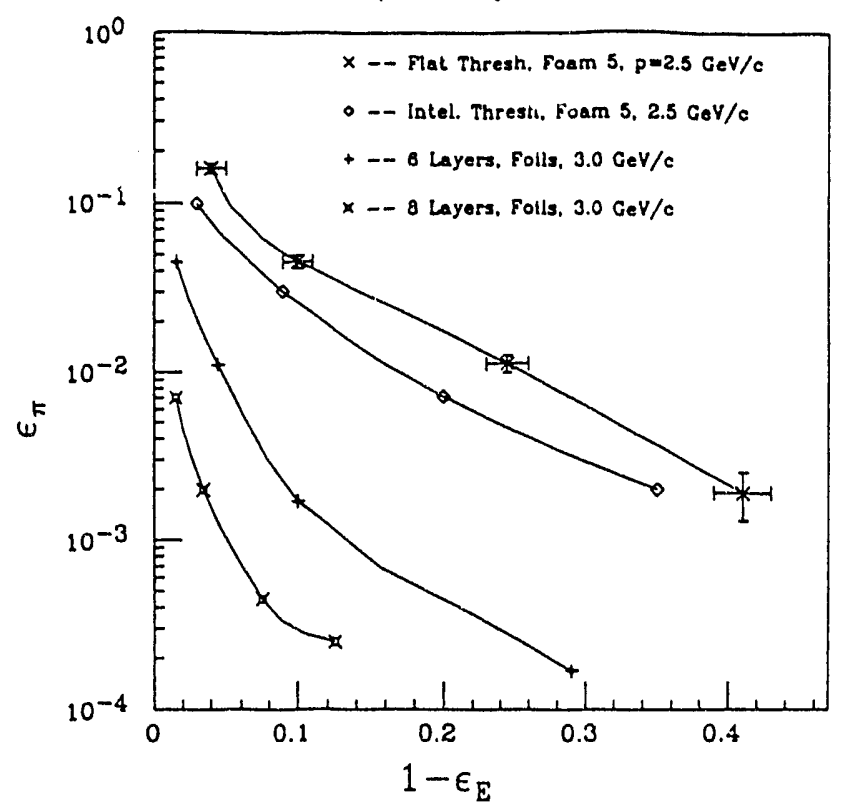

Figure 6: Pion rejection vs. electron efficiency for 2.5 and $3.0 \mathrm{GeV} / \mathrm{c}$ particles. Curves shown are for 8-layer foam radiators with simple threshold, 8 layer foam and intelligent threshold, 6 layer foils and 8 layer foils each with intelligent threshold

ter location moves away from the radiator. The intelligent threshold is effective because $\delta$-rays from pions are evenly distributed in the $z$ direction (beam direction) inside the drift volume while transition radiation photons are not. Lower energy TR photons tend to be absorbed earlier in the $\mathrm{Xe}$ gas, that is closer to the radiator, whereas higher energy $T R$ photons penetrate farther into the gas detector before being absorbed.

Results of the analysis of data taken at both 2.5 and $3.0 \mathrm{GeV} / \mathrm{c}$ can be seen in figure 6 . This figure shows four curves:(a) $e / \pi$ rejection for 8 TRD planes using foam radiator and a simple threshold cut,(b) the same detectorradiator combination and an intelligent threshold,(c) 6 TRD planes using foil radiator and the intelligent threshold, and (d) 8 TRD foil radiator planes and the intelligent threshold. One sees that with 8 layers of foam radiator and $90 \%$ electron efficiency one retains $3 \times 10^{-2}$ pions. Likewise, $90 \%$ electron efficiency and 6 or 8 planes of foil radiator retain $2 \times 10^{-3}$ and $3 \times 10^{-4}$ pions respectively. We conclude that the foil radiators will easily provide us with the desired $e / \pi$ rejection above $2.0 \mathrm{GeV} / c$, but that the foam radiators needs further study if they are to be used in a RHIC experiment. Mechanical construction of the TRD would be considerably easier if one used foam radiators rather than foil radiators, but it is still possible to construct a large area TRD with foil radiators.

\section{$\mathrm{dE} / \mathrm{dx} \quad e / \pi$ Separation}

Below a momentum of $2.5 \mathrm{GeV} / \mathrm{c}$ the effectiveness of $e / \pi$ separation using $T R$ photons starts to diminish because

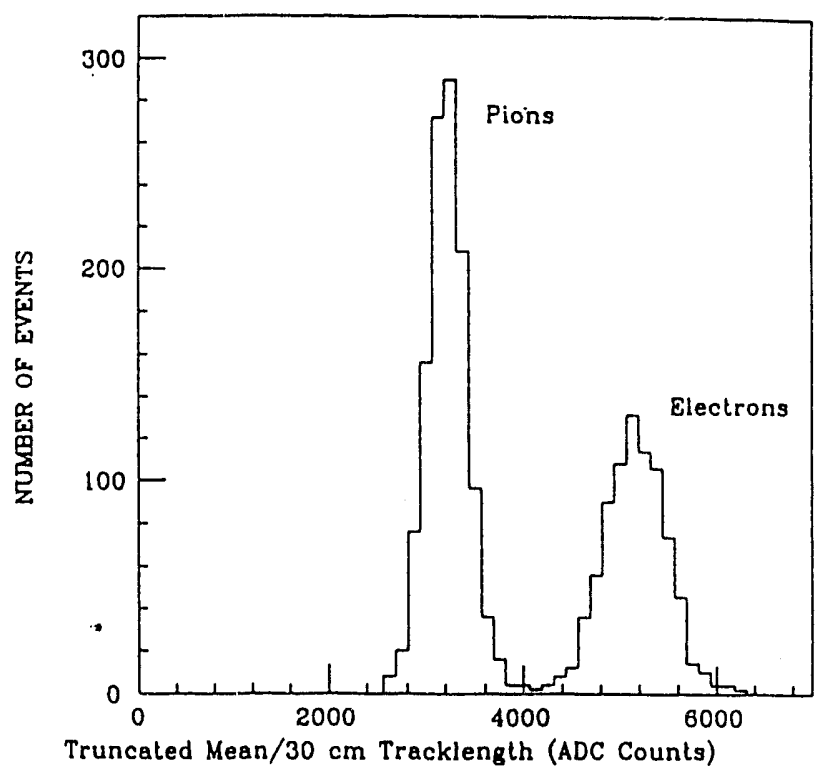

Figure 7: Electron, pion $\mathrm{dE} / \mathrm{dx}$ distribution for $30 \mathrm{~cm}$ track length and $60 \%$ truncated mean. The data is for particles with momenta $1.0 \mathrm{GeV} / \mathrm{c}$ and $35^{\circ}$ incident angle.

as the electron becomes less relativistic, the probability of a TR photon being emitted by the radiator decreases. Since it is desirable to have good $e / \pi$ separation below this momentum, other methods must be considered. Particle ID using $\mathrm{dE} / \mathrm{dx}$ in the Time-Expansion Chamber can be used to achieve good $e / \pi$ separation below $2.5 \mathrm{GeV} / \mathrm{c}$.

In the momentum region $200 \mathrm{MeV} / \mathrm{c}$ to $3.0 \mathrm{GeV} / \mathrm{c}$, an electron loses more energy through $\mathrm{dE} / \mathrm{dx}$ than a pion $\mathrm{due}$ to its position on the relativistic rise of the $d E / d x$ curve as described by the Bethe-Bloch formula. Energy losses in xenon gas are large, so a low momentum track traveling through a sufficient path length of xenon can be identified as either an electron or a less relativistic particle by evaluating the truncated means of the $\mathrm{dE} / \mathrm{dx}$ distributions. The average charged track moving through the RHIC TRD passes through $30 \mathrm{~cm}$ of xenon gas as it crosses the eight layers of the detector. In our analysis of the test data we have combined multiple pion or electron events to obtain the equivalent $30 \mathrm{~cm}$ track length in the xenon gas to evaluate the $\mathrm{dE} / \mathrm{dx} \quad e / \pi$ rejection. The $\mathrm{dE} / \mathrm{dx}$ data have been taken at three angles $\left(0^{\circ}, 20^{\circ}, 35^{\circ}\right)$ and with two different gas mixtures $\left(95 \% \mathrm{Xe}+5 \% \mathrm{C}_{4} \mathrm{H}_{10}, 50 \% \mathrm{Xe}+45 \% \mathrm{He}+\right.$ $5 \% \mathrm{C}_{4} \mathrm{H}_{10}$ ).

The drift velocity in the test TRD is $\sim 20 \mathrm{~mm} / \mu \mathrm{sec}$ which translates to a drift distance of $0.5 \mathrm{~mm}$ per 24.5 nsec FADC time bins. If one takes each FADC time bin as an independent measurement, one obtains $600 \mathrm{ADC}$ data samples for each $30 \mathrm{~cm}$ charged track. We combined events containing either electrons or pions until we obtained data equivalent to a $30 \mathrm{~cm}$ track length. We created a pulse area(PA) distribution of the $600 \mathrm{FADC}$ values/track, eliminated the highest $40 \%$ of the FADC values and calculated the mean PA value of the remainder. This is the standard 


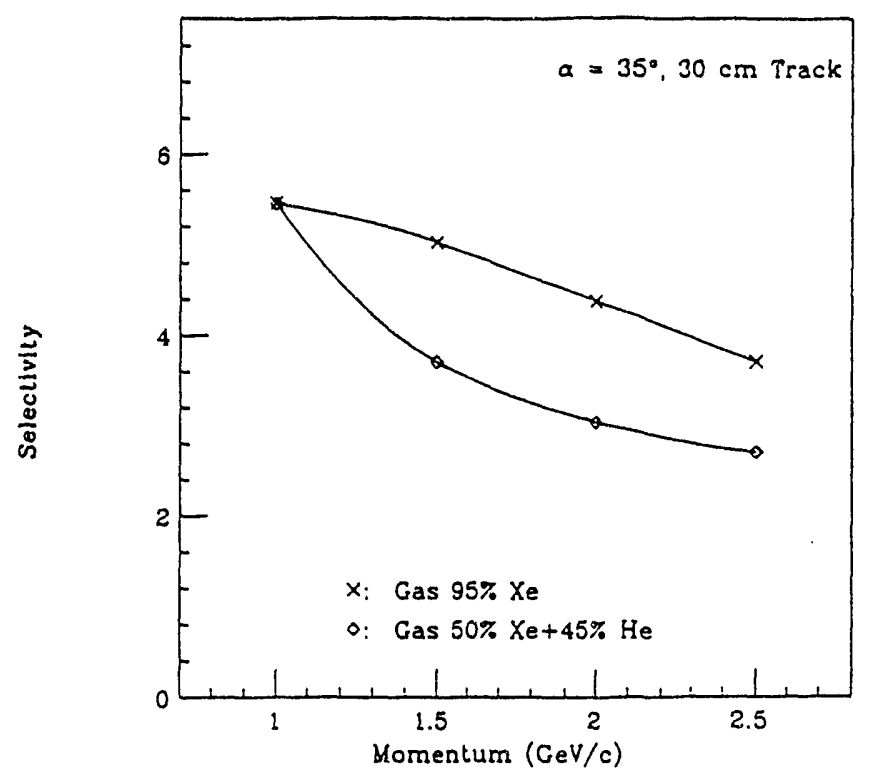

Figure 8: S-value for 2 different gas mixtures. $95 \% \mathrm{Xe}+$ $5 \% \mathrm{C}_{4} \mathrm{H}_{10}$ and $50 \% \mathrm{Xe}+45 \% \mathrm{He}+5 \% \mathrm{C}_{4} \mathrm{H}_{10}$. The data is for $30 \mathrm{~cm}$ track length and $35^{\circ}$ incident angle.

truncated-mean technique. A distribution of these mean PA values shows a large separation between the mean PA of electrons and pions (Figure 7). The electron-pion separation shown in figure 7 can be described by the selectivity value (S-value) where:

$$
S=\frac{\left(E_{e}-E_{\pi}\right)-2 \sigma_{e}\left(E_{\pi} / E_{e}\right)}{\sigma_{\pi}}
$$

$E_{\varepsilon}$ and $E_{\pi}$ represent the means of the $\mathrm{dE} / \mathrm{d} x$ truncated mean distribution for electrons and pions.

Figure 8 shows the $\mathrm{S}$-value plotted versus momentum as obtained from our $35^{\circ} \mathrm{dE} / \mathrm{d} x$ data. Our results agree with that of previous studies $[3,4]$. The S-value is larger for data taken with the TRD filled with a higher percentage of xenon, which is expected since a larger percentage of xenon yields larger $\mathrm{dE} / \mathrm{d} x$ losses.

The $e / \pi$ separation in figure 9 shows that for $35^{\circ}$ tracks at $1.5 \mathrm{GeV} / \mathrm{c}$, one has $97 \%$ electron efficiency while keeping only $1.5 \times 10^{-3}$ pions. The average track angle in the RHIC TRD is predicted to be $35^{\circ}-40^{\circ}$. The $e / \pi$ separation in our data has a strong dependance on track angle. The dependance is understood to be due to space charge building up along the anode wires, which effectively reduces the chamber gas gain for the electron clusters drifting in at times later in the avalanche process. One expects that this effect could be ameliorated with a careful optimization of the gas mixture and detector gain.

It is important to understand how the ADC resolution effects the $\mathrm{dE} / \mathrm{dx} \quad e / \pi$ separation so that the minimum number of ADC bits required to do the job can be determined. The resolution of our 6-bit FADC was artificially reduced in our analysis to make the data appear as if it had come from an ADC with a fewer number of bits. The result of this procedure shows that the S-value improves

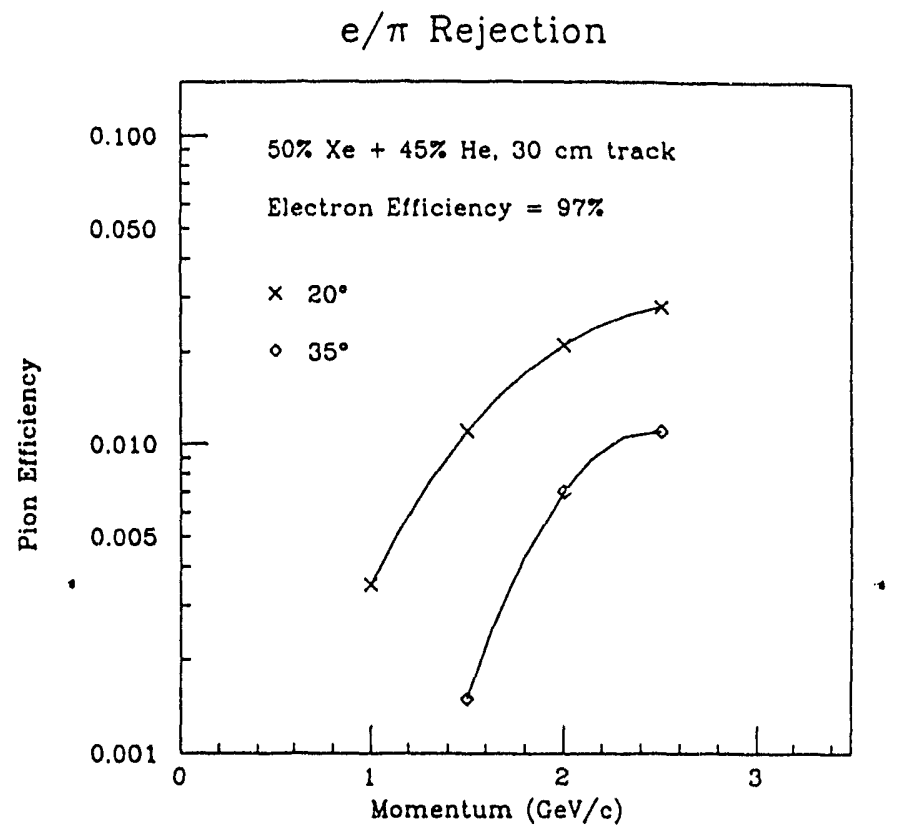

Figure 9: $\mathrm{dE} / \mathrm{dx} e / \pi$ rejection for $97 \%$ electron efficiency and $50 \% \mathrm{Xe}+45 \% \mathrm{He}+5 \% \mathrm{C}_{4} \mathrm{H}_{10}$ gas. The track length is $30 \mathrm{~cm}$ and the incident angles are $20^{\circ}$ and $35^{\circ}$.

\section{dli: /dx S-Value vs liADC Bil.s}

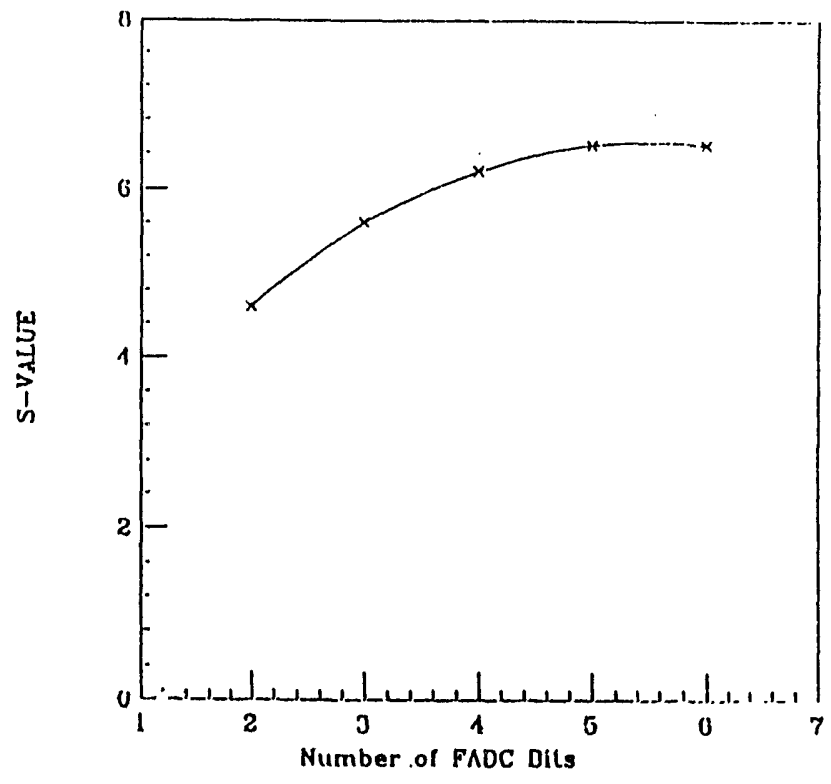

Figure 10: S-value for $\mathrm{dE} / \mathrm{dx} e / \pi$ rejection vs. effective number of FADC bits 
Test TRD - Anode Position Resolulion

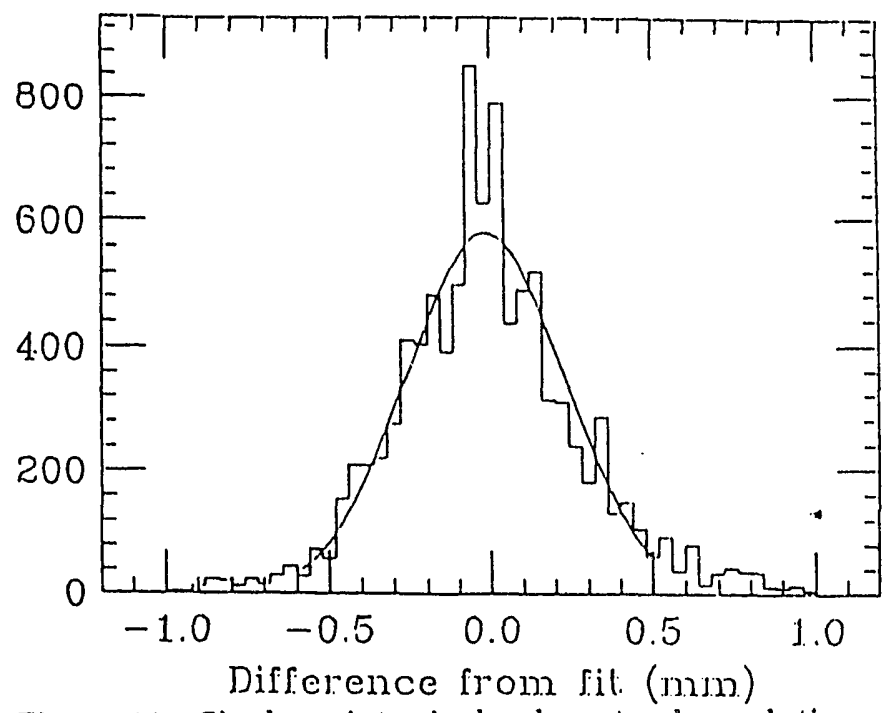

Figure 11: Single point, single plane track resolution of $230 \mu \mathrm{m}$ RMS using TRD anode wires, $0^{\circ}$ track angle.

with $A D C$ resolution until one reaches 4-bits, after which the S-value increases only marginally (Figure 10).

\section{Track Reconstruction}

Instrumenting the TRD with FADC electronics allows one to not only do $e / \pi$ separation using $\mathrm{dE} / \mathrm{dx}$ techniques but to also track all charged particles through the detector. Our RHIC experiment requires the momentum of each charged track in the detector to be reconstructed with a $\Delta p / p=0.2 \%$ at $1.0 \mathrm{GeV} / \mathrm{c}$. GEANT calculations show that to achieve this one requires a single point resolution of $\sim 250 \mu \mathrm{m}$ RMS. Reconstructing 4-plane detector tracks in our test data using a simple $\chi^{2}$ algorithm yields a single point resolution of $230 \mu \mathrm{m}$ RMS for $0^{\circ}$ tracks and $200 \mu \mathrm{m}$ RMS for $35^{\circ}$ tracks (Fig. 11). Since the geometry and FADC clock cycle for both the prototype and our RHIC TRD are quite similar, we expect the position resolution for the test TRD to be about the same as that of the final TRD.

\section{Summary}

We have conducted a test run with a TRD that has the ability to do particle ID using both transition radiation and $d E / d x$ measurements, while also tracking all charged particles through the detector with a single point resolution better than $250 \mu \mathrm{m}$ RMS. We have tested a variety of polyethylene foam radiators and found that, when normalized to a constant thickness in radiation lengths, they produce on average $50 \%$ less $\mathrm{TR}$ photons than polypropylene foil radiators. Results from foam radiator tests show that we can expect an $e / \pi$ rejection of $3 \times 10^{-2}$ for 8 planes of TRD and $p \geq 2.5 \mathrm{GeV} / c$, while the equivalent foil radiator would yield a rejection of $3 \times 10^{-4}$. The $e / \pi$ rejection using $\mathrm{dE} / \mathrm{dx}$ run from $1.5 \times 10^{-3}$ for $35^{\circ}$ tracks at $1.5 \mathrm{GeV} / \mathrm{c}$ to $1 \times 10^{-2}$ at $2.5 \mathrm{GeV} / \mathrm{c}$. The $\mathrm{dE} / \mathrm{dx}$ rejection is a function of both xenon content in the gas and track angle, however the dependance on track angle may be reduced with optimization of the gas mixture. The particle ID and tracking ability of the TRD we have tested is well matched to the performance requirements for detectors at RHIC.

\section{Acknowledgments}

We gratefully acknowledge the technical assistance of August Hoffmann. We would like to thank Bo Yu for his assistance with the figures and the analysis. We would also like to thank our AGS liaison engineer Dave Dayton, and the rest of the AGS staff.

\section{References}

[1] S. Nagamiya et al., RHIC OASIS experiment Letter of Intent, Brookhaven National Laboratory, unpublished.

[2] M. Clemen et al., Proc. of Symposium on Particle ID at High Luminosity Hadron Colliders, FNAL(1989),339.

[3] P. Rehak and A. Walenta,IEEE Trans. Nucl. Sci. NS-27, pp.54(1980).

[4] T. Ludlam et al., LEEE Trans. Nucl. Sci. NS-28, pp.439(1981). 


\section{DISCLAIMER}

This report was prepared as an account of work sponsored by an agency of the United States Government. Neither the United States Government nor any agency thereof, nor any of their employees, makes any warranty, express or implied, or assumes any legal liability or responsibility for the accuracy, completeness, or usefulness of any information, apparatus, product, or process disclosed, or represents that its use would not infringe privately owned rights. Reference herein to any specific commercial product, process, or service by trade name, trademark, manufacturer, or otherwise does not necessarily constitute or imply its endorsement, recommendation, or favoring by the United States Government or any agency thereof. The views and opinions of authors expressed herein do not necessarily state or reflect those or the United States Government or any agency thereof. 

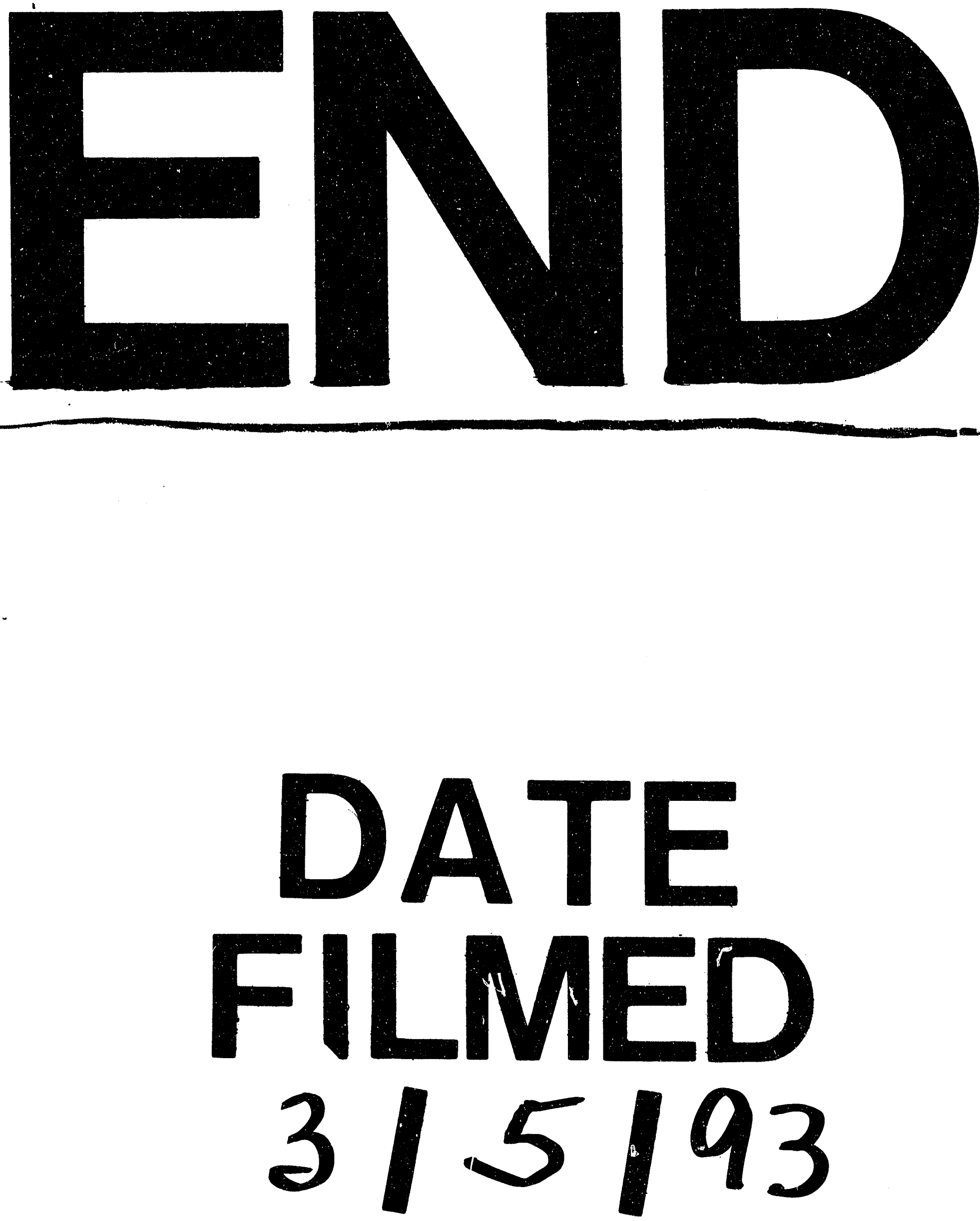
